\title{
Direct Visualization of Pyrrole Reactivity upon Confinement within a Cyclodextrin Metal-Organic Framework
}

\author{
Alejandro Nuñez-Lopez, Marta Galbiati, Natalia M. Padial, Carolina R. Ganivet, Sergio Tatay, \\ Emilio Pardo, Donatella Armentano, and Carlos Martí-Gastaldo*
}

\begin{abstract}
Metal-organic frameworks can be used as porous templates to exert control over polymerization reactions. Shown here are the possibilities offered by these crystalline, porous nanoreactors to capture highly-reactive intermediates for a better understanding of the mechanism of polymerization reactions. By using a cyclodextrin framework the polymerization of pyrrole is restricted, capturing the formation of terpyrrole cationic intermediates. Single-crystal $X$-ray diffraction is used to provide definite information on the supramolecular interactions that induce the formation and stabilization of a conductive array of cationic complexes.
\end{abstract}

$M$ etal-organic frameworks (MOFs) are crystalline, porous materials built from the interconnection of either metal ions or clusters and organic linkers to produce porous architectures. MOFs found rapid application in gas storage and separation thanks to their unlimited chemical/structural versatility combined with a fine control over their porosity metrics. ${ }^{[1]}$ Compared to other porous materials like layered clays, zeolites, sol-gel matrices, or polymers, these metalorganic scaffolds also offer important advantages when used as functional hosts to encapsulate molecules. ${ }^{[2]}$ For instance, as beautifully illustrated by the pioneering work of Kitagawa, Uemura and co-workers, ${ }^{[3,4]}$ the micro/nanoporous channels in these materials can be tailored to template the docking of monomers and confine polymerization reactions. In this way, the size, shape, and morphology of the empty channels, together with their dimensionality and surface chemistry, can be effectively used to gain control over the molecular weight, stereoregularity, composition, or sequence of the resulting polymers. $^{[5]}$

These works confirm the direct influence of MOFs over polymerization reactions when used as templates, either from restricting the packing of monomer guests to a confined

[*] A. Nuñez-Lopez, Dr. M. Galbiati, Dr. N. M. Padial, Dr. C. R. Ganivet, Dr. S. Tatay, Dr. E. Pardo, Dr. C. Martí-Gastaldo

Instituto de Ciencia Molecular (ICMol), Universitat de València Paterna 46980, València (Spain)

E-mail: carlos.marti@uv.es

Dr. N. M. Padial

Current address: Department of Chemistry

The Scripps Research Institute

10550 North Torrey Pines Road, La Jolla, CA 92037 (USA)

Dr. D. Armentano

Dipartimento di Chimica e Tecnologie Chimiche, Università della Calabria, 87030, Cosenza (Italy)

(2) Supporting information and the ORCID identification number(s) for (iD the author(s) of this article can be found under:

https://doi.org/10.1002/anie.201904890. nanospace or by chemical interaction with the groups decorating the surface of the pores. However, these experiments are generally limited to microcrystalline powders and do not enable direct visualization of monomer reactivity inside the channels of the MOF. As result, the structural information on the chemical and geometrical principles that dictate the assembly of the polymer for specific packing modes, local environments, or conformations is often missing. This information is in turn inferred indirectly from the properties of the resulting polymers. We argued that translating this same concept to single-crystal studies would be ideal for evaluating, unambiguously, the effect of the supramolecular interactions or geometrical restrictions (pore size and shape) imposed by the framework over the chemical reactivity of loaded monomers. Our work goes one step further in this regard and demonstrates the possibilities of MOF crystalline nanoreactors to capture highly-reactive intermediates for a better understanding of the mechanism of polymerization reactions. We make use of a cyclodextrin (CD) MOF, Rb-CD-MOF (or CD-MOF-2), to control the reactivity of redox-active pyrrole (Py) monomers by confining its chemical polymerization to three-dimensional (3D) nanochannels. Compared to uncontrolled polymerization in solution, the use of a framework as a host-guest template restricts the mobility and reactivity of pyrrole by supramolecular pinning of the confined monomers to enable the formation and stabilization of terpyrrole (Tpy) cations.

$\mathrm{Rb}-\mathrm{CD}-\mathrm{MOF}$ can be prepared as millimeter-sized, colorless crystals by direct reaction of $\mathrm{RbOH}$ and $\mathrm{CD} \cdot{ }^{[6]} \mathrm{Rb}-\mathrm{CD}$ MOF crystals have already proven to be robust enough to retain their original crystallinity upon impregnation with molecular guests or formation of metal nanoclusters inside their channels. ${ }^{[7-9]}$ This robustness was expected to simplify the crystallographic study and physical characterization. Previous work also confirms the ability of CDs to stabilize
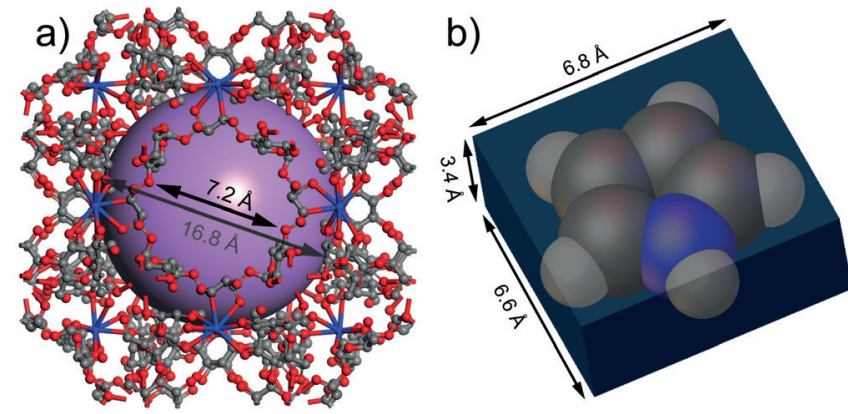

Figure 1. a) Structure of Rb-CD-MOF featuring mesoporous cavities of $16.8 \AA$ (purple sphere) interconnected within the solid by pore windows of $7.2 \AA$ b) Dimensions of pyrrole. See Table S1 for details. 
Py molecules by forming supramolecular inclusion complexes that can be then used as precursors for chemical polymerization. ${ }^{[10]}$ As shown in Figure 1, the structure of Rb-CD-MOF is based on the interconnection of $(\gamma-\mathrm{CD})_{6}$ units that adopt a body-centered cubic packing. This packing results in the intersection of 1D channels in the three crystallographic directions with internal cavities and pore windows of 16.8 and $7.2 \AA$, respectively, big enough to accommodate Py molecules $(6.8 \AA \times 6.6 \AA \times 3.4 \AA) .{ }^{[6]}$ We expected this $3 \mathrm{D}$ porous architecture to avoid the diffusion impediments intrinsic to $1 \mathrm{D}$ and 2D dimensionalities.

$\mathrm{Rb}-\mathrm{CD}-\mathrm{MOF}$ crystals were synthesized by vapor diffusion of $\mathrm{MeOH}$ in an aqueous solution of $\mathrm{RbOH}$ and $\gamma-\mathrm{CD}$, with slight modifications to the reported procedure. ${ }^{[6]}$ As-made crystals were thoroughly washed with $\mathrm{MeOH}$ and exchanged with $\mathrm{CH}_{2} \mathrm{Cl}_{2}$. We used anhydrous solvents to avoid damage to the crystals, which were then stored in a glove box prior to use. Figure 2 summarizes the steps followed to study the reactivity of Py after MOF loading. Commercial grade pyrrole was

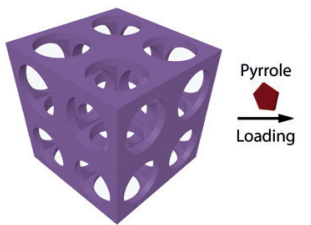

$\mathrm{Rb}-\mathrm{CD}-\mathrm{MOF}$

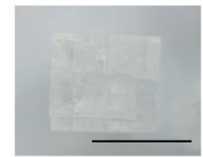

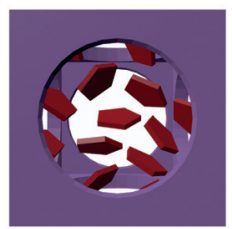

Rb-CD-MOFCPy

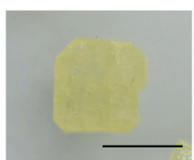

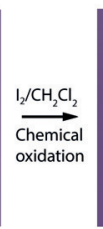

Rb-CD-MOFCPy(ox)

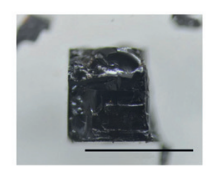

Figure 2. Schematic representation of the loading and chemical oxidation of pyrrole monomers within Rb-CD-MOF mesopores. The bottom panel shows the color changes in the crystals of the MOF for the different stages of the process. Scale bars correspond to $250 \mu \mathrm{m}$.

distilled under vacuum (see Figure S1 in the Supporting Information), protected from light, and stored under an inert atmosphere. Solvent exchanged Rb-CD-MOF crystals were immersed at room temperature under static conditions in a pyrrole $/ \mathrm{CH}_{2} \mathrm{Cl}_{2}$ solution ( $30 \% \mathrm{v} / \mathrm{v}$ ) for 72 hours. The loading was carried out in the glove box by using freshly distilled Py and anhydrous solvents to prevent partial oxidation. The excess Py was removed by thorough washing in $\mathrm{CH}_{2} \mathrm{Cl}_{2}$. Loading of the monomer was preliminarily identified by the change in color of the crystals, from colorless to yellow, consistent with the presence of a few of the representative bands of Py in the FT-IR spectrum (see Figure S2). Rb-CD$\mathrm{MOF} \subset \mathrm{Py}$ was fully dissolved in $\left[\mathrm{D}_{6}\right] \mathrm{DMSO}$. The ${ }^{1} \mathrm{H}$ NMR spectra confirm the presence of $\mathrm{CD} \subset \mathrm{Py}$ inclusion complexes in solution with an upfield shift of $0.038 \mathrm{ppm}$ in $\mathrm{H}_{\mathrm{b}}$ and $\mathrm{H}_{\mathrm{e}}$ protons and $0.066 \mathrm{ppm}$ in $\mathrm{H}_{\mathrm{c}}$ and $\mathrm{H}_{\mathrm{d}}$ protons of $\mathrm{Py}$, compared to the free monomer, as a result of the chemical interaction with the CD (see Figure S3). ${ }^{[10]}$ We estimated a total content of close to 12 Py molecules per unit formula of Rb-CD-MOF using NMR spectroscopy (see Figure S4). This value is quite close to the theoretical uptake capacity calculated by Monte Carlo in Materials Studio R2.
Previous work reported that polypyrrole (PPy) can be synthesized in situ by oxidative polymerization reaction of Py monomers loaded into $\mathrm{Cu}^{\mathrm{II}}$ and $\mathrm{Fe}^{\mathrm{III}}$ redox-active frameworks $^{[11,12]}$ In our case, soaking Rb-CD-MOF $\subset$ Py in organic solutions of these metals at variable concentrations always led to uncontrolled oxidation and irreversible damage to the crystals with complete loss of crystallinity. We discarded this route and chose to use a mild oxidant, such iodine, as an auxiliary oxidizing agent. This two-step strategy has proven to be more respectful with the crystallinity of inclusion compounds upon chemical oxidation. ${ }^{[13,14]}$ Accordingly, freshly prepared $\mathrm{Rb}-\mathrm{CD}-\mathrm{MOF} \subset \mathrm{Py}$ crystals were soaked in a solution of iodine in dichloromethane $(0.05 \mathrm{M})$ at $4{ }^{\circ} \mathrm{C}$ for 72 hours. Whereas reaction at $4^{\circ} \mathrm{C}$ proceeded with no changes to the size or shape of the crystals (Figure 2), when the same experiment was conducted at room temperature we identified significant morphological damage likely resulting from the more favorable conditions for the polymerization reaction. After 24 hours, chemical oxidation in the inclusion compound was evidenced by the black color of $\mathrm{Rb}-\mathrm{CD}-\mathrm{MOF} \subset \mathrm{Py}(\mathrm{ox})$ crystals. The reaction was maintained for 72 hours and the excess iodine in $\mathrm{Rb}-\mathrm{CD}-\mathrm{MOF} \subset \mathrm{Py}(\mathrm{ox})$ was washed off several times with aliquots of fresh solvent.

For a first analysis of the product of the reaction, we attempted to dissolve the crystals in $\left[\mathrm{D}_{6}\right] \mathrm{DMSO}$. They formed a black solid upon dissolution and it was isolated by filtration. ${ }^{1} \mathrm{H}$ NMR spectra of the solution only displays signals from $\gamma$ $\mathrm{CD}$, confirming the absence of unreacted pyrrole molecules (see Figure S5). The solid-state ${ }^{13} \mathrm{C}$ magic-angle spinning (MAS) NMR spectrum of the solid confirms the presence of a broad $\alpha$ carbon signal at $\delta=128.5 \mathrm{ppm}$, indicative of the formation of cationic polypyrrole (see Figure S6). ${ }^{[13]}$ The ${ }^{13} \mathrm{C}$ $\mathrm{CP}-\mathrm{MAS}$ spectrum of $\mathrm{Rb}-\mathrm{CD}-\mathrm{MOF} \subset \mathrm{Py}(\mathrm{ox})$ crystals is dominated by $\gamma$-CD with a very feeble signal associated with the product of the oxidation, preventing more detailed analysis of host-guest interactions (see Figure S7). ${ }^{[14]}$ The thermogravimetric analysis (TGA) of Rb-CD-MOF before and after the pyrrole loading displays a weight loss $(6 \%)$ at $180^{\circ} \mathrm{C}$, below that corresponding to the decomposition of the framework, indicative of the filling of the pores with organic molecules. $\mathrm{Rb}-\mathrm{CD}-\mathrm{MOF} \subset \mathrm{Py}(\mathrm{ox})$ presents a higher mass loss at low temperatures because of the sublimation of iodide and also shows a significant mass loss at higher temperatures that can be linked to the presence of occluded iodide in the crystals that form RbI, which sublimates close to $650^{\circ} \mathrm{C}$ (Figure $3 \mathrm{~b}$ ). $\mathrm{N}_{2}$ adsorption isotherms were used to confirm the effect of Py loading and oxidation over the porosity of the material (Figure 3c). Rb-CD-MOF displays a BET surface area of $882 \mathrm{~m}^{2} \mathrm{~g}^{-1}$, consistent with the reported value, ${ }^{[6]}$ that is reduced to $286 \mathrm{~m}^{2} \mathrm{~g}^{-1}$ after Py impregnation in Rb-CD$\mathrm{MOF} \subset \mathrm{Py}$. In turn, chemical oxidation leads to complete loss of porosity probably because of the polymerization of Py in the pores combined with the inclusion of iodide anions formed during the oxidation. The analysis of the pore-size distribution (PSD) by using density-functional theory (DFT) shows that the cumulative pore volume corresponding to the mesoporous cages in the MOF centered at $1.72 \mathrm{~nm}$, drop from 0.342 to $0.137 \mathrm{~cm}^{3} \mathrm{~g}^{-1}$ after Py loading and down to $0.006 \mathrm{~cm}^{3} \mathrm{~g}^{-1}$ after exposure to $\mathrm{I}_{2}$. PSD changes confirm that 
a)

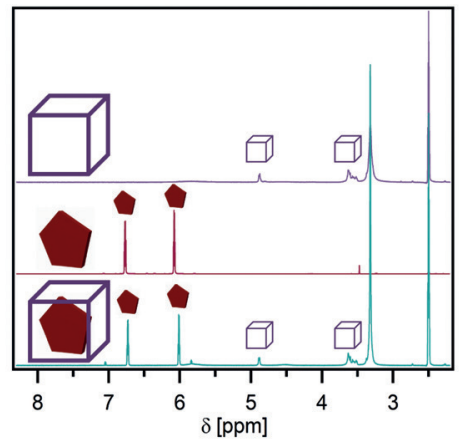

b)

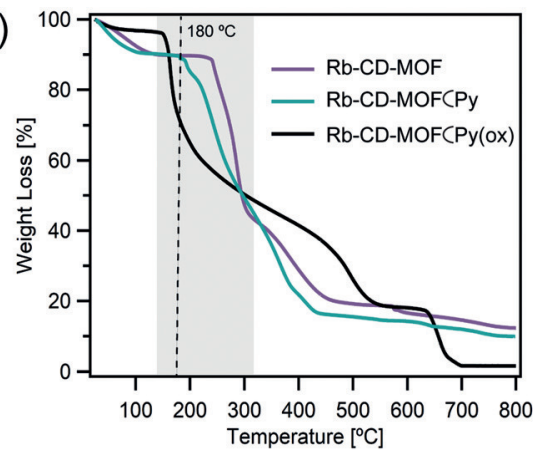

c)

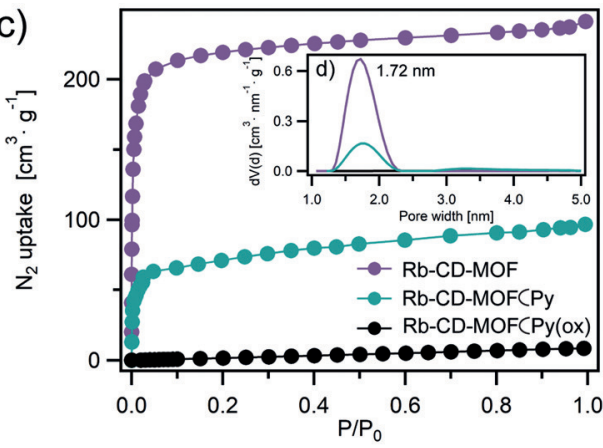

Figure 3. a) ' $\mathrm{H}$ NMR spectra of Rb-CD-MOF (purple), distilled pyrrole (maroon), and Rb-CD-MOFCPy (green). b) TCA profiles and c) $\mathrm{N}_{2}$ adsorption isotherms of Rb-CD-MOF, Rb-CD-MOF $\subset$ Py and Rb-CD-MOF $\subset$ Py(ox) (black). d) Pore-size distribution using density-functional theory of (c).

the fraction of empty space available for the diffusion of the oxidant in $\mathrm{Rb}-\mathrm{CD}-\mathrm{MOF} \subset \mathrm{Py}$ is lost after the reaction (Figure $3 \mathrm{~d})$.

For a better understanding of the mechanism controlling the oxidative polymerization of confined Py, we next studied crystals of Rb-CD-MOF $\subset \mathrm{Py}(\mathrm{ox})$ by using single-crystal X-ray diffraction (SC-XRD). As shown in Figure 4, the structure of $\mathrm{Rb}-\mathrm{CD}-\mathrm{MOF}$ is robust enough to resist the oxidation of pyrrole with $\mathrm{I}_{2}$ and undergoes a single-crystal to single-crystal (SCSC) transformation. In good agreement with our analysis of the bulk, density maps and structural analyses confirm the oxidation of the included Py to generate Rb-CD-MOF $\subset$ Tpy, that combines cationic terpyrrole (TPy) units and $\mathrm{I}_{3}{ }^{-}$anions in the pores of the solid (Figure $4 \mathrm{a}$; see Figure S10). In accordance with BET and PSD analysis, the channels are almost entirely filled by these ionic molecular guests that account for close to $92 \%\left(13464.3 \AA^{3}\right)$ of the empty volume available. Similar to previous crystallographic studies analyzing confined chemical transformations in MOFs, ${ }^{[15,16]}$ the guest molecules suffer from a certain structural disorder that was restrained in the refinement (see the Supporting Information for details).
Rb-CD-MOF $\subset$ Tpy retains the chiral $I 432$ space group intrinsic to $\mathrm{Rb}-\mathrm{CD}-\mathrm{MOF}$, that is built from the interconnection of $(\gamma-\mathrm{CD})_{6}$ units and $\mathrm{Rb}^{\mathrm{II}}$ centers to conform a 3D solid with a body-centered cubic packing (Figure $4 \mathrm{a}$ ). Crystals of $\mathrm{Rb}-\mathrm{CD}-\mathrm{MOF} \subset \mathrm{Tpy}$ are isomorphous to the parent material confirming the preservation of the network after loading and chemical oxidation. Unlike other indirect techniques, SCXRD enables direct visualization of the formation of [TPy ${ }^{+}$ cations formed by the oxidative coupling of three Py units through 2,5-positions of the heterocycle. This intermediate is consistent with the mechanism generally accepted for the formation of polypyrrole by electropolymerization. ${ }^{[17]}$ However, the formation of such trimers during chemical oxidative polymerization reactions was unclear until now. [TPy $]^{+}$ cations and counterbalancing $\mathrm{I}_{3}{ }^{-}$anions occupy the large spherical pores at the center of each $(\gamma-C D)_{6}$ cube, and are stabilized by either ionic or supramolecular interactions (Figure $4 \mathrm{~b}, \mathrm{c}$ ). The quality of the diffraction data evidence that, even though the crystallinity is maintained upon oxidative SCSC, $[\mathrm{TPy}]^{+}$molecules inherit a statistical disorder between two positions symmetrically related (Figure $4 \mathrm{~d}$ ). It is worth noting that the uncertainty of the X-ray analysis makes a)

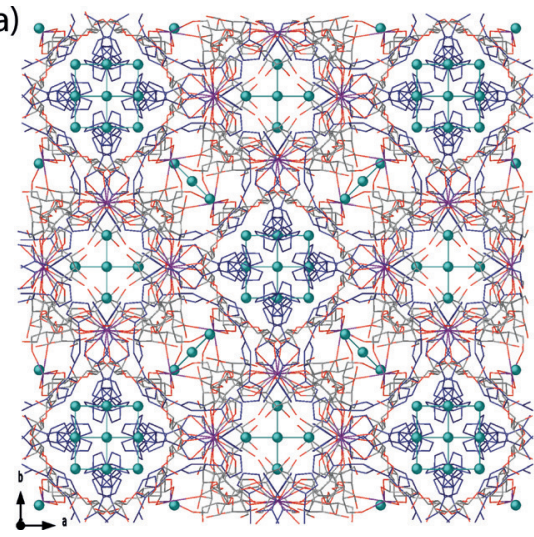

b)

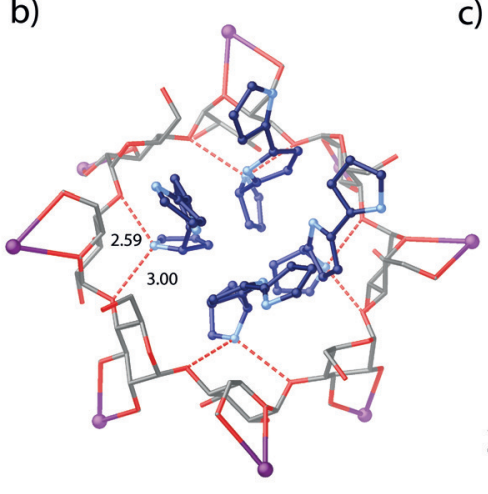

c)

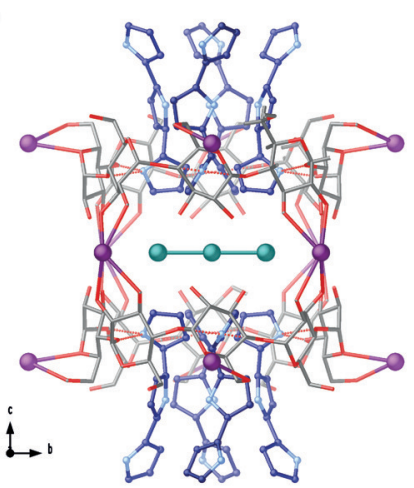

d)

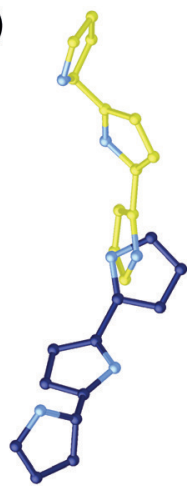

Figure 4. Views of different sections of the structure of Rb-CD-MOF $\subset$ Tpy determined by single-crystal X-ray diffraction. a) View along the crystallographic $c$ axis of a portion of the 3D Rb-CD-MOF $\subset$ Tpy crystal structure. b) Perspective views along the crystallographic $c$ axis of the approximately spherical pores, located at the center of each $(\gamma-C D)_{6}$ cube, filled by [TPy] ${ }^{+}$cations with details of host-guest interactions. c) Perspective view along the crystallographic $a$ axis of the $[\mathrm{TPy}]^{+}$and $\mathrm{I}_{3}^{-}$moieties. d) Perspective view of the observed crystallographic disorder for $[\mathrm{TPy}]^{+}$cations. Rubidium, nitrogen, and iodine atoms are represented by purple, sky blue, and green spheres, respectively. Carbon atoms of the $[\mathrm{TPy}]^{+}$guests are depicted as blue spheres, whereas oxygen and carbon atoms from $\gamma$-cyclodextrin ligands are simplified as red and grey sticks. Free water solvent molecules are omitted for clarity. d) The yellow carbon atoms in the figure highlight the two possible orientations of the guests. 
it difficult to exclude the formation of pentapyrrole $\left[\mathrm{Py}_{5}\right]^{+}$ oligomers (with positional disorder on the central ring) even though $[\mathrm{TPy}]^{+}$units are by far the most probable resolution due to several reasons (see Crystallographic section in the Supporting Information). This ambiguity was ruled out with MALDI-TOF after digestion of the crystals, by confirming the presence of a strong peak at $m / z 199.11$ in excellent agreement with the formation of cationic terpyrrole (see Figure S8). The high reactivity of this cation in solution prevented its quantification by NMR spectroscopy (see Figure S9). The two possible orientations of $[\mathrm{TPy}]^{+}$are linked to the framework and involve the formation of bifurcated hydrogen bonds between $\mathrm{NH}$ groups of the terminal Py unit and the acetal oxygen bridges from the primary face of $\gamma$-CD with $\mathrm{N} \cdots \mathrm{O}$ distances of 2.59(2) and 3.00 (2) A (Figure 4b,c; see Figure S10b). Compared to this same interaction in the inclusion compound $\alpha-\mathrm{CD} / \mathrm{Py}, 3.16-$ (4) $\AA{ }^{[10]}$ the shorter distances in Rb-CD-MOF $\subset$ Tpy suggest more polar interactions for a stronger stabilization of the guest. This network of host-guest hydrogen-bond interactions, together with the limited number of confined Py molecules, is possibly the main reason for the restriction of the polymerization of Py. The formation of $4[\mathrm{TPy}]^{+}$cations per unit cell in $\mathrm{Rb}-\mathrm{CD}-\mathrm{MOF} \subset \mathrm{TPy}$ agrees well with the 12 Py units estimated by NMR analysis for Rb-CD-MOF $\subset \mathrm{Py}$, confirming that the final product is formed exclusively by reorganization of the monomers loaded. A control experiment was used to show that free $\gamma$-CD could not produce similar results (S6). This difference is striking compared to other porous hosts like mesoporous zeolites ${ }^{[18]}$ or microporous MOFs based on aromatic linkers, ${ }^{[11,12,19,20]}$ for which oxidation always proceeds until polypyrrole is formed, with changes to its dimensionality and polydispersity modulated by the template. In this case, the incorporation of $\mathrm{CD}$ rings to the surface of the channels provides a periodic distribution of conical cavities with a hydrophobic interior rich in $\mathrm{OH}$ hydrogen-bond-acceptor groups. These recognition sites are very effective in restraining the mobility and reactivity of the encapsulated Py monomers, which can only polymerize to a limited degree for the formation of the reactive intermediate $[\mathrm{TPy}]^{+}$, which is also stabilized in the channels by supramolecular interactions. $\mathrm{I}_{3}{ }^{-}$molecular anions also display statistical disorder with some iodine atoms $\left(\mathrm{I}_{2}\right.$ and $\left.\mathrm{I}_{4}\right)$ modelled on two sites with a half each other occupancy factors (see Figures S10-S12). The average bond length within each crystallographically distinct $\mathrm{I}_{3}{ }^{-}$anion, $2.85 \AA$, agrees well with the value reported. ${ }^{[21]}$

The impregnation and oxidative polymerization of pyrrole or thiophene is an effective strategy to endow insulating MOFs with extrinsic electrical conductivity, simply by backfilling the pores with the conducting polymers generated in situ. ${ }^{[11,12,19,20,22]}$ Oxidation generally leads to morphological damage that results in polycrystalline samples that are often amorphous. As a result, previous works on extrinsically conductive MOFs make use of pressed pellets to collect electrical measurements. However, Rb-CD-MOF crystals are hundreds of micrometers wide and robust enough to withstand SCSC transformation and can be directly contacted to minimize the influence of grain boundaries in the conductivity measurements. To investigate the effect of short-range polymerization over the conductivity of the inclusion compound, we measured the electrical conductivity of Rb-CDMOF, $\subset$ Py, and $\subset$ TPy single crystals by using a two-probe contacts method (Figure 5a).

Single crystals of the three materials were manually contacted to a chip by using Pt wires and conductive silver paint and measured at a base pressure of 7 torr in a physical properties measurements system (PPMS; Figure 5b). The first set of data collected proved the importance of using a controlled water-free atmosphere to collect reproducible measurements. Figure 5c shows a comparison of the $I-V$ curves collected for all the crystals up to $4 \mathrm{~V}$. As expected, Rb$\mathrm{CD}-\mathrm{MOF}$ and $\mathrm{Rb}-\mathrm{CD}-\mathrm{MOF} \subset \mathrm{Py}$ behave as insulators with conductivities in the range of $10^{-12}-10^{-11} \mathrm{Scm}^{-1}$. In turn, Rb$\mathrm{CD}-\mathrm{MOF} \subset \mathrm{TPy}$ displays a million-fold enhancement of the electrical conductivity up to $5 \times 10^{-6} \mathrm{~S} \mathrm{~cm}^{-1}$. Previous works on the oxidative synthesis of polymers by using MOFs as templates report a broad dispersion of conductivity values between $10^{-6}$ and $10^{-1} \mathrm{Scm}^{-1}$, depending on the dimensionality of the polymer and the characteristics of the MOF. ${ }^{[10,18,19]}$ The conductivity of $\mathrm{Rb}-\mathrm{CD}-\mathrm{MOF} \subset \mathrm{TPy}$ lies close to that
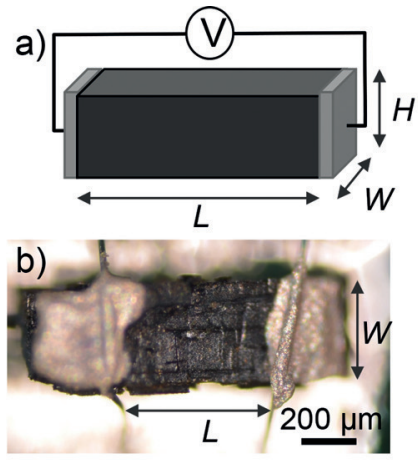
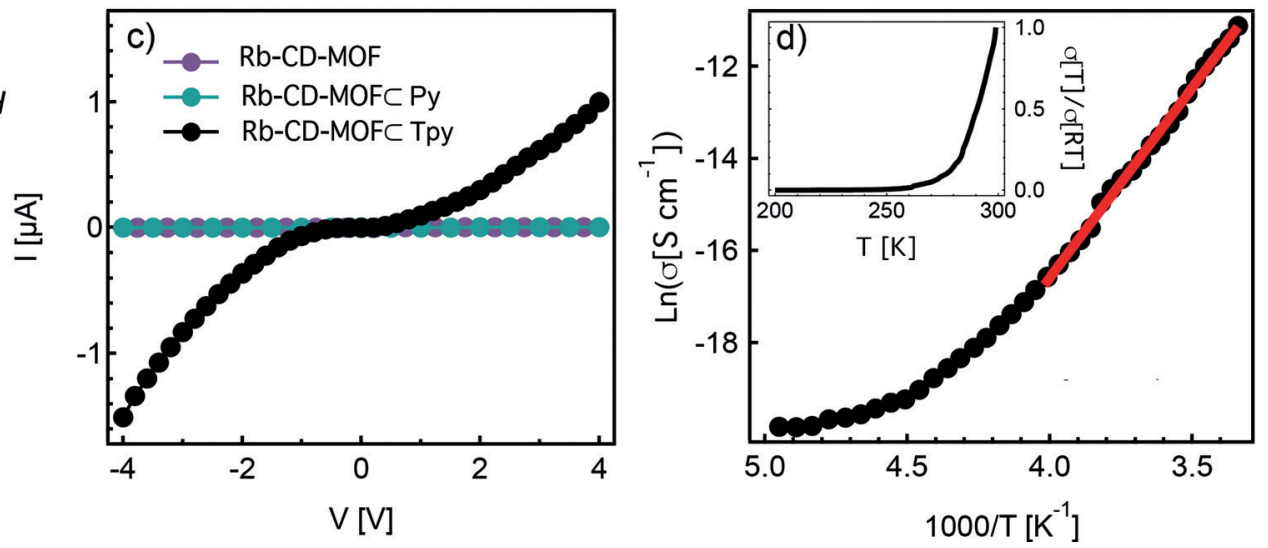

Figure 5. a) Scheme of electrical measurements in a two-probe configuration. b) Optical microscope image of a single crystal of Rb-CD-MOF $\subset$ Tpy

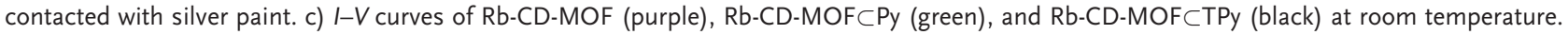
d) $\operatorname{Ln}(\sigma(\mathrm{T}) / \sigma(\mathrm{RT}))$ of Rb-CD-MOF $\subset$ Tpy plotted as a function of the inverse of the temperature at a bias voltage of $1 \mathrm{~V}$. Conductivity was calculated by estimating dimensional parameters from optical microscope images and approximating the crystal as rectangular structures. The activation energy $\left(E_{\mathrm{a}}\right)$ was calculated from the slope of the linear fit at room temperature. Inset shows the $\sigma(T) / \sigma(R T)$ as a function of temperature. 
reported for 2D PPy arrays growth in a redox-active layered coordination polymer. ${ }^{[10]}$ Figure $5 \mathrm{~d}$ shows the thermal dependence of the conductivity with temperature measured at a bias voltage of $1 \mathrm{~V}$. The exponential decrease of conductivity with temperature suggests that $\mathrm{Rb}-\mathrm{CD}$ MOF $\subset$ TPy displays a semiconductive behavior. According to the Arrhenius law, the linear fit of $\ln (\sigma(T) / \sigma(R T))$ to the reciprocal of the temperature in the high-temperature range yields a $E_{\mathrm{a}}$ value of $709 \mathrm{meV}$. This value is considerably higher than the $0.2 \mathrm{eV}$ reported for highly-oriented 1D PPy, for which unusually high conductivity was ascribed to percolating conducting paths with the templating framework, ${ }^{[19]}$ but comparable to that observed for electron tunneling between metal nanoclusters spatially separated in CD-MOF. ${ }^{[7]}$ This charge-transport mechanism is possibly the origin for the electrical conductivity of the segregated iodine terpyrrole complexes formed in Rb-CD-MOF $\subset$ TPy.

In summary, we have used a cyclodextrin framework to control the chemical oxidation of pyrrole by confined reactivity. Compared to other MOFs based on $\pi$-rich aromatic linkers that lead to the formation of polypyrrole, interaction of the guest with the $\mathrm{CD}$ rings in the framework restrict the polymerization of pyrrole to a low degree for the formation and stabilization of a conductive array of cationic terpyrrole complexes. Our work offers unprecedented structural snapshots for definite information on the host-guest interactions that control this process by using SC-XRD. We are confident these findings will contribute to gain control over the translation of MOF surface chemistry into controlled reactivity of confined redox-active molecules for targeted polymeric assemblies with either controllable physical properties or even advanced functions.

\section{Acknowledgements}

This work was supported by the EU (ERC Stg Chem-fs-MOF 714122) and Spanish MINECO (CTQ2017-83486-P, RYC2012-10894, RYC-2016-19817 and MDM-2015-0538). A.N.-L. thanks the Spanish MECD for FPU predoctoral grant (FPU16/02220). N.M.P. thanks the Marie Skłodowska-Curie Global Fellowships (749359-EnanSET). We thank XALOC beamline staff at ALBA Synchrotron for their help with preliminary experiments. D.A. thanks the Italian MIUR (Ministero dell'Instruzione, dell'Università e della Ricerce) for the "Fondo per il finanziamento delle attività base di ricerca". Ismael Mullor-Ruíz, Gemma Peñalver, Neyvis Almora-Barrios, and Isabel Solana are also acknowledged.

\section{Conflict of interest}

The authors declare no conflict of interest.

Keywords: metal-organic frameworks . noncovalent interactions - oxidation - structure elucidation . supramolecular chemistry
How to cite: Angew. Chem. Int. Ed. 2019, 58, 9179-9183 Angew. Chem. 2019, 131, 9277-9281

[1] H. Furukawa, K. Cordova, M. O’Keeffe, O. M. Yaghi, Science 2013, 341, 1230444.

[2] J. Juan-Alcañiz, J. Gascon, F. Kapteijn, J. Mater. Chem. 2012, 22, $10102-10118$.

[3] T. Uemura, N. Yanai, S. Kitagawa, Chem. Soc. Rev. 2009, 38, $1228-1236$.

[4] T. Uemura, S. Horike, S. Kitagawa, Chem. Asian J. 2006, 1, 3644.

[5] T. Uemura, S. Mochizuki, T. Kitao, Chem. Commun. 2018, 54, $11843-11856$

[6] R. Smaldone, R. Forgan, H. Furukawa, J. Gassensmith, A. Slawin, O. Yaghi, J. Stoddart, Angew. Chem. Int. Ed. 2010, 49, 8630-8634; Angew. Chem. 2010, 122, 8812-8816.

[7] S. Han, S. C. Warren, S. Yoon, C. D. Malliakas, X. Hou, Y. Wei, M. G. Kanatzidis, B. A. Grzybowski, J. Am. Chem. Soc. 2015, 137, 8169-8175.

[8] S. Han, Y. Wei, B. A. Grzybowski, Chem. Eur. J. 2013, 19, $11194-$ 11198.

[9] R. S. Forgan, R. A. Smaldone, J. J. Gassensmith, H. Furukawa, D. B. Cordes, Q. Li, C. E. Wilmer, Y. Y. Botros, R. Q. Snurr, A. M. Slawin, et al., J. Am. Chem. Soc. 2012, 134, 406-417.

[10] J. Storsberg, H. Ritter, H. Pielartzik, L. Groenendaal, $A d v$. Mater. 2000, 12, 567-569.

[11] N. Yanai, T. Uemura, M. Ohba, Y. Kadowaki, M. Maesato, M. Takenaka, S. Nishitsuji, H. Hasegawa, S. Kitagawa, Angew. Chem. Int. Ed. 2008, 47, 9883-9886; Angew. Chem. 2008, 120, $10031-10034$.

[12] T. Uemura, Y. Kadowaki, N. Yanai, S. Kitagawa, Chem. Mater. 2009, 21, 4096-4098.

[13] G. Street, T. Clarke, M. Krounbi, K. Kanazawa, V. Lee, P. Pfluger, J. Scott, G. Weiser, Mol. Cryst. Liq. Cryst. 1982, 83, 253 264.

[14] A. Comotti, S. Bracco, M. Mauri, S. Mottadelli, T. Ben, S. Qiu, P. Sozzani, Angew. Chem. Int. Ed. 2012, 51, 10136-10140; Angew. Chem. 2012, 124, $10283-10287$.

[15] F. R. Fortea-Pérez, M. Mon, J. Ferrando-Soria, M. Boronat, A. Leyva-Pérez, A. Corma, J. Herrera, D. Osadchii, J. Gascón, D. Armentano, E. Pardo, Nat. Mater. 2017, 16, 760-766.

[16] M. Mon, F. Lloret, J. Ferrando-Soria, C. Martí-Gastaldo, D. Armentano, E. Pardo, Angew. Chem. Int. Ed. 2016, 55, 1116711172; Angew. Chem. 2016, 128, 11333-11338.

[17] J.-C. Lacroix, F. Maurel, P.-C. Lacaze, J. Am. Chem. Soc. 2001, 123, 1989-1996.

[18] T. Bein, P. Enzel, Angew. Chem. Int. Ed. Engl. 1989, 28, $1692-$ 1694; Angew. Chem. 1989, 101, 1737-1738.

[19] Q. Wang, C. Zhang, Macromol. Rapid Commun. 2011, 32, 1610 1614.

[20] B. Dhara, S. S. Nagarkar, J. Kumar, V. Kumar, P. Jha, S. K. Ghosh, S. Nair, N. Ballav, J. Phys. Chem. Lett. 2016, 7, $2945-$ 2950.

[21] H. Wang, J. Ge, C. Hua, C. Jiao, Y. Wu, C. F. Leong, D. M. D'Alessandro, T. Liu, J. Zuo, Angew. Chem. Int. Ed. 2017, 56, 5465-5470; Angew. Chem. 2017, 129, 5557-5562.

[22] T. Uemura, R. Nakanishi, S. Mochizuki, S. Kitagawa, M. Mizuno, Angew. Chem. Int. Ed. 2016, 55, 6443-6447; Angew. Chem. 2016, $128,6553-6557$.

Manuscript received: April 19, 2019

Accepted manuscript online: May 3, 2019

Version of record online: May 28, 2019 